\title{
ON ARTIFICIAL MODIFICATION OF LIGHT REACTIONS AND THE INFLUENCE OF ELECTROLYTES ON PHOTOTAXIS
}

\author{
WOLFGANG F. EWALD
}

Since Loeb's early papers on animal heliotropism it has been known that in certain cases the experimentor is able to bring about changes in the orientation of animals to light by artificial means. Loeb was able to show that in marine copepods decrease of temperature and increase of concentration of the seawater made negatively phototactic animals positive, and vice versa: that increase of temperature and decrease of concentration made positive animals negative. Similar effects of temperature were noted in Polygordius larvae. Strassburger ('78), Massart, Holmes and Mast found the opposite effects in swarm-spores, Protozoa and a species of Ranatra. Later on Loeb supplemented his communications by the discovery, that freshwater Gammarus, Daphnia and Volvox could be made strongly positive by adding acids, especially $\mathrm{CO}_{2}$, to the water. The same clear results could not, however, be obtained in marine planktonic forms. In the nauplius of Balanus perforatus Loeb could observe a strong influence on phototaxis only by exposing the animals to very strong or very weak light. Strong light made positive animals negative, weak light made negative animals positive. The ultra-violet rays proved to be specially effective in making positive animals negative. In fact, their effect was stronger than that of all other rays combined. Rothert, in experimenting on the effect of alcohol, ether and chloroform on free swimming plants, found the light reactions of Gonium and Pandorina to be inhibited by some of these narcotics in a certain dosation, the power of locomotion remaining unaffected.

According to the investigations of Tappeiner and Hertel a similar modification of light reactions is brought about by cer- 
tain dyes, especially by eosine. Hertel showed, that Paramaecia and Rotatoria can be killed by light of certain wave-lengths, provided that by artificial staining their protoplasm is put in a position to absorb the light rays acting upon it. Ultra-violet light, however, obtained from the spectrum of magnesium-sparks, had a deadly effect also on unstained animals and was thereby shown to be absorbed by protoplasm under all circumstances. Green light, for instance, would attain the same result only, after eosine was added to the water containing the animals, in a dilution of 1:1200. This effect of certain dyes, because of its similarity to the process of color-sensitisation in photography, has been defined as 'sensitisation' of the protoplasm. According to Hertel, it is also possible to make the retractor-muscle of the syphon in Sipunculus directly sensitive to light by staining it with eosine 1:3000, while the ventral cord in the same species is stated to be sensitive to light without staining, through natural pigmentation.

Starting from these experiments I tried to obtain modifications of the light reactions in various marine animals by artificial means. I must confess that my expectation to find changes in the sensibility of a given species to colored rays of not excessive intensity by staining the animals, was not fulfilled. Nevertheless, I hope that the results obtained in the other experiments mentioned may justify publication.

My experiments were carried on in a spacious dark room of the Naples Zoölogical Institute from February to August 1911. I wish to express my special obligations to the Prussian Ministry of Education for the use of the table and to Dr. Burian, Dr. Bauer and Dr. Cerutti for their help and advice during the course of my work.

After some weeks of unsuccessful research the nauplii of Balanus perforatus, on which Groom and Loeb had experimented more than twenty years ago, proved to be the most satisfactory objects. These authors have thoroughly investigated and described the characteristic behavior of the nauplii. After hatching, the larvae show strong positive phototaxis. After being exposed to light for some time they first begin to oscillate between 
the positive and negative orientation and then finally reverse their reaction, becoming negative with a velocity proportionate to the light intensity. If the light is very weak, the reversion does not occur at all. As the authors saw positive animals swim from direct sunlight into diffused daylight and negative animals take the opposite course, provided only that they were following the direction of the light rays, they concluded, that, in accordance with Loeb's theory of phototaxis, not light intensity, but the direction of the light rays, was the determining factor in orientation. ${ }^{1}$ Moreover they did not succeed in finding any influence of sudden changes of intensity on the behavior of the nauplii. They concluded from experiments with red and blue glass, that the short wave-lengths were of greater effect than the long ones. Finally, they tried the effect of changes in temperature and salt-concentration, but without apparent results. Hess, in the course of investigation on the sense of sight in invertebrate animals, arrived at the conclusion, that they all showed the maximum of stimulation in the green and yellow-green parts of the spectrum, not in the blue and violet, as was to be expected by the older experiments with color-screens. This caused Loeb to reinvestigate the question, together with Maxwell. They experimented on the Californian Balanus and Volvox following the method of Hess. After placing a cuvette containing the animals, in the light of a spectrum, they found the largest gathering in the green. Hess simultaneously obtained similar results with Balanus at Naples.

I first examined the behavior of the nauplii in white light of a 50 c. p. electric lamp. They were seen to become negative much more rapidly on the first day after hatching, than on the following. From day to day their sensitiveness to light slowly decreased. Brought from the dark into light they all show a characteristic reaction, sinking to the bottom more or less quickly, according to the intensity of illumination and collecting there

\footnotetext{
${ }^{1}$ On the evidence offered this conclusion was since shown to have been drawn without cogent reason (Hess, Ewald) and the antithesis of 'intensity' and 'direction' not to be justified in this form (Ewald, Mast) though the underlying idea was a fruitful one and a step forward.
} 
on the side facing the lamp. If the lamp is attached at a level with the animals, outside the glass containing them, they mostly remain near the bottom and but a small column of nauplii is seen along the side up to the surface. Not before the lamp is lifted considerably above the surface level does the aggregation of nauplii near the surface increase. If one now inserts a smoked glass between lamp and glass vessel, all the animals instantaneously rise towards the surface, but some of them will sink again after some time. By inserting a second smoked glass the same effect may be attained a second time, causing practically all animals to collect near the surface. If one now takes away one of the smoked glass panes, all nauplii will begin rising again for just a moment, but soon commence sinking. One observes a column of animals moving down fairly rapidly and finally stopping; gradually they begin reascending. Obviously the nauplii of Balanus show a typical reaction to changes of intensity of illumination, just as I was able to demonstrate it for Cladocera and Copepods. Increase of illumination (within certain limits) causes first slight acceleration, then inhibition of locomotion, making the animal sink. Decrease of illumination causes acceleration of locomotion, making the animal move toward the source of light. The same absolute light intensity will cause the 'negative reflex' when following a weaker illumination and the 'positive reflex' when following a stronger one. This shows the nauplii of Balanus to adapt themselves to different light intensities, provided these intensities remain unchanged for a sufficient time. If one brings the source of light vertically above the glass vessel, one does not generally succeed in observing the sinking movement after increase of illumination. The animals collect near the surface and their locomotion, directed vertically upwards, is sufficient to keep them there even after a decrease in the energy of locomotion. With the light coming from the side the locomotion is directed horizontally, and even small changes in the rate of locomotion will result in a conspicuous change of position.

I now tried to find out which wave lengths of the spectrum had the strongest influence on the reactions to changes of intensity of illumination described above. Positively phototactic animals 
were distributed in eight glass tubes of $12 \mathrm{~mm}$. diameter, arranged side by side in a small stand. A spectrum obtained from an arc lamp by means of a carbon bisulphide prism, was thrown obliquely from above on the row of glasses. Before beginning the experiment I made the animals, who were collected near the surface, adapt themselves to weak light reflected from the ceiling. As soon as the arc lamp was switched on, the animals began to sink in the green and yellow-green, after that in the blue-green, blue and yellow and finally in the violet and the red. The lower border of the part of the tubes filled with animals formed a blunt cone having its lowest point in the green and yellow-green. The same experiment could also be made in the reverse way. I made the animals adapt themselves to the different spectrum colors. When a strong white light was switched on at some distance from the side of the glasses, the animals in the green part of the spectrum would sink last, as they were adapted to the strongest light, those in the red and violet first.

By another experiment it can be shown, that the same rays that bring about the strong reactions to changes of the intensity of illumination have also the strongest orienting power. If a narrow cuvette with parallel sides, filled with larvae, is exposed to the spectrum, the long axis being cut at right angles by the direction of the rays, the major part of the animals accumulates in the yellow-green and green. One can observe the animals swimming toward this part from all sides, leaving the rest of the glass nearly free. In the yellow-green they gradually sink to the bottom and collect there in large numbers near the front pane. The negative animals are found in the violet and red near the rear pane, while the green part remains free of negative animals. These experiments, frequently repeated, confirm the results obtained by Hess, who found the maximum of reaction to light in a great number of invertebrates to occur in the green and yellow-green parts of the spectrum. It is important to keep in mind, that the same rays have the strongest effect on the reactions to changes of intensity of illumination described above. I find it necessary to state in parenthesis that the ani- 
mals in being oriented by the light rays follow a line strictly defined by the parallelogram of forces. An animal swimming on one side of a trough covered by the light of a spectrum, say, in the violet, will be directed both by the violet rays coming from the source of light and by the blue, green and yellow-green light dispersed by the front pane of the trough, as can be seen with the naked eye. The animal may consequently be observed to direct its course between the two, arriving at the front pane, say, in the blue. It now continues to work along against the pane, but directed obliquely against the green part, till it comes to rest in the green light itself and is now oriented chiefly by the direct light. Even a very slight excess of stimulation on one side by the dispersed green rays will suffice to bring animals utlimately into the green. Loeb is therefore perfectly justified in assuming that the phototactic animal moves in the direction of the light rays and statements to the contrary made by different authors since the beginning of experiments on animal tropisms, lastly by Hess ${ }^{2}$ as recently as 1911, must be based either on insufficient observation or on inaccurate reasoning. It is necessary to come to a plain understanding on this question after so many years of experiments.

Having ascertained these facts it was important to know whether the green rays possessing the strongest effect on phototactic motor reflexes would also be most important for the process of making positive animals negative. This question cannot by any means be answered in the affirmative a priori, as previous authors have tacitly done. If it is probable that the

2 Hess's assumption that phototactic animals are not forced to move along the line of the light rays but choose their way to the field of strongest illumination--at right angles to the direction of the light rays if necessary - is due to his overlooking the facts mentioned above and to a faulty interpretation of the tropism theory. The tropism theory does not assume the animals to move towards 'the' source of light, unless there is really one source of light only. If light can strike the animal from several points, even if the excess of light on one side be very slight, it follows a course defined by the parallelogram of forces, as has been pointed out by Loeb from the very beginning. It is Loeb's merit, to have pointed out the purely machine-like and stereotyped character of phototactic reactions which differ from orientation in higher vertebrates by the very fact that there never can be any 'choice' in the direction of locomotion. 
reactions to changes of intensity described above and shown to be instantaneous and very easily reversible, are effected through the medium of the eyes, this is not certain for the relatively slow non-adaptive process of negativation. Should this process be brought about by photic stimulation of the eye, we would have to assume either a second slower, non-adaptive photochemical process running alongside of the other, or else summatory action of the quick and adaptive process, leading to a new and stronger effect. These conclusions seem cogent, after the adaptive character of the reactions to changes of intensity of illumination has been recognized.

A fact which may at first sight speak against the photoreceptors as mediators of the process of negativation is the discovery made by Loeb, that ultra-violet light of a mercury lamp makes the Balanus-larvae negative at a quicker rate, than sunlight, especially if the latter is deprived of its ultra-violet rays by means of a glass plate. The rays of shortest wave-length would thus have a stronger negativating influence than all the other rays put together. This maximum would not coincide with that found for orientation. On the other hand, it is possible that in the case of the ultra-violet rays we are dealing with a special case influenced by the deleterious effect of these rays on the entire protoplasm; that ultra-violet light acts directly on the chemical processes of metabolism and stands apart in its effect. It is shown by the following experiment, that ultraviolet light has a strongly deleterious effect also on the Balanuslarvae.

Two blackened watch glasses containing animals were put in diffused daylight or sunlight. One was covered with a strong. clean glass plate, the other was left uncovered. After a few minutes in sunlight or one to two hours in strong diffused light the nauplii in the uncovered dish were killed. Those in the covered dish lived for hours. I have often repeated this experiment. Every time a simple glass plate, which did not weaken the visible rays ostensibly sufficed to retard the harmful effect of strong light on the nauplii, showing thereby that the effect 
was due to the ultra-violet rays. The glass plate had also a visibly retarding effect on the negativating process.

Returning to the effect of light on the process of negativation, I will first give a description of the visible phenomena connected with this process. The animals normally collect near the surface on the side of the vessel nearest to the light ('positive pole' of the vessel) provided the rays strike the vessel obliquely or from the side. If the light intensity is not inframinimal for negativation, the animals begin after some time to sink to the bottom and to collect there likewise at the positive pole. It is not before a considerable time that the second phase of the process begins, the nauplii reversing their orientation and, in consequence, collecting round the negative pole. According to the light intensity the first phase will last for a longer or shorter span of time.

To decide the effect of the different wave lengths of visible light on the process of negativation I proceeded by two different methods. First I used an arrangement similar to that described above for testing the effect of different wave lengths on the phototactic reflexes and orientation. The animals, equally divided in thirteen small tubes, were brought into the strong primary spectrum of a Rowland grating. Sunlight, projected into the dark room by means of a heliostat, was used for illumination. When the glass tubes were brought from the dark into the light of the spectrum, one could again observe the animals sinking most strongly in the green, less so in the blue, still less in the yellow, while they remained close to the surface in the red and the violet. This difference was observed to persist, if the light rays struck the glasses about horizontally, this being a case of permanent regulation of the position by light intensity. If the nauplii were not too old-younger than forty-eight hours-they would begin to become negative after some time, first in the green, shortly after in the violet. Later on the animals in the blue-green and blue followed, finally those in the yellow. In the red part only a very slight negativating effect could be observed. One glass served as a control and was placed outside the spectrum. Here the animals would not become negative. As soon as the animals became 
negative, they were removed from the glasses with a pipette. As a consequence, the tubes in the violet and the green light were first emptied, all nauplii having become negative; the blue and the yellow tubes followed much later, and the red would take a very long time.

Similar results were obtained with color-filters. The same glass tubes which had been disposed in the spectrum were put into wider glasses containing a colored liquid. The glass tubes were corked and entirely surrounded by the liquid. The filterliquids consisted for

Violet of methyl-violet (area transmitted from $400-470 \mu$ and from $640-700 \mu$ )

Blue of copperacetate + methyl-violet with addition of ammonia (area transmitted from $445-485 \mu$ )

Green of copperacetate + potassium monochromate with addition of ammonia (area transmitted from $480-560 \mu$ )

Red of lithium carmine (area transmitted from $610-700 \mu$ )

The layer of colored liquid surrounding the glasses was nowhere less than $12 \mathrm{~mm}$. deep. The transmitted areas were tested with a Zeiss spectroscope and as far as possible the extinction of all the areas was made equal by means of the Engelmann 'Microspectralphotometer;' that is to say: each area was made to trarsmit the same fraction of the colored light of equal wave lengths existing in the solar spectrum by regulating the saturation of the colored solution.

As in the previous experiments, the different position of the animals in each color after distribution in the different glasses was very noticeable; as usual the nauplii were nearest the bottom in the green, nearest the surface in red and violet. If the sensitiveness of the larvae stood in a favorable proportion to the intersity of illumination, it was frequently observed, that they became negative in the violet and green only and remained positive for several hours in all other colors. If I then brought the glasses into full sunlight, the animals became negative also in the blue and finally in the red light. Brought back into diffused daylight they became positive in the reverse order. The experiments with color-filters are, however, not as conclusive as the others, owing to the impossibility of separating with 
sufficient exactitude the effect of the extinction of the monochromatic light from the effect of its wave length. In fact the effect varied, especially in the green, according to the opacity of the solution.

We may state in conclusion, that two maxima of negativating effect were found inside the visible part of the spectrum: one in the green and one in the violet, the minimum being in the red. If we were to show the negativating effect of light rays by a curve, we should have to begin with a maximum in the ultra-violet slowly falling towards the blue, rising again to the yellow-green and falling steadily towards the minimum in the red. The nature of the curve makes it probable, that we have to deal with at least two interacting effects, one of which may operate by the medium of the eyes (maximum in the green) while the other acts on some body substances through the cuticle directly (maximum in the ultra-violet).

I cannot, however, refrain from mentioning the fact, that this result was not always attained. Especially during experiments in which the pure, strong and broad spectrum of the Rowland grating was replaced by the considerably smaller, weaker and less pure spectrum of a carbon bisulphide prism, the effect in the green was invariably so small, that I stated a minimum instead of a maximum in this part. The negativating influence of the yellow and even the red rays was sometimes stronger than that of the green ones, whereas the effect on the motor reflexes was also in this case strongest in the green.

To conclude, I will mention a few experiments on the deleterious effect of concentrated monochromatic light. If the animals contained in the color-filter glasses described above were exposed for about two hours to full sunlight, they were killed first in the violet, later in the green and the blue and not at all in the red. To the eye the red solution seemed most transparent, the violet nearly opaque. The permeability to ultraviolet rays was probably equally small in all glasses.

I shall now proceed to describe a series of experiments carried out in order to determine the effect of variations of the temperature and the chemical composition of the water on the light 
reactions of Balanus-larvae. Before describing these experiments, it should be mentioned that my results were always controlled by comparison with animals, caught at the same pole of the same vessel as the animals experimented on, but kept in pure seawater of normal temperature. This is important, as the animals change their reaction when exposed to light without any other influence being brought to bear on them, and as it is therefore possible only to determine the influence of the agent to be tested by comparison with normal animals under equal conditions of illumination (and temperature). I will mention first the effect of changes in temperature.

I found without exception, that increase of temperature made positive animals negative and negative animals more negative, and that decrease of temperature made negative animals positive and positive animals more positive. I never noted any uncertainty in this effect of temperature. The amount of the change in temperature necessary for the reversing of the reaction to light depends on the age and state of the larvae. The relation between the inclination to positive or negative reaction, the quotient $\frac{p}{n}$ indicating the degree of neutralisation of two antagonistic processes causing positive or negative reaction, constitutes what is called the 'Lichtstimmung' of the organism. The factor of 'Lichtstimmung' must be considered in all observations on the effect of stimulating agents. If, in our case, the nauplii are approaching the negative condition owing to the effect of illumination, a slighter increase in light intensity will effect the change of reaction than if they had just been exposed to light. Young animals are more easily influenced than old ones. A change in temperature of about 5 or $6^{\circ} \mathrm{C}$, however, always has the desired effect. At about $+5^{\circ} \mathrm{C}$. the nauplii fall into cold rigor; they will stand being treated to more than $30^{\circ} \mathrm{C}$. As an increase of about $1^{\circ} \mathrm{C}$. will under certain circumstancesespecially if the larvae are newly hatched-suffice to make them negative, it is most important to maintain a constant temperature during all experiments on phototaxis. 
I will next discuss the influence of the salts of the seawater on phototaxis. In this connection I desire to express my thanks to to Dr. O. Meyerhof, who gave me many valuable hints. Acting on his advice I used 0.65 molecular solutions which corresponded best to the concentrations found in the Naples Aquarium water at the time. I made up solutions of chemically pure sodium chloride, potassium chloride, magnesium chloride, magnesium sulphate and calcium chloride. The magnesium and calcium chloride were tested by titration. The effects of the different salts were studied firstly by adding them to pure isotonic sodium chloride solution, secondly by adding them to natural seawater and finally by eliminating successively each component salt from artificial seawater made up of the five components mentioned, with the addition of the necessary amount of alkali. The artificial seawater had the following composition:

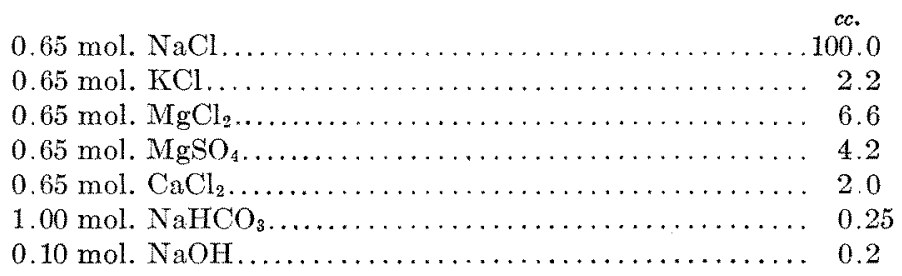

The amount of alkali is that found by Warburg at the Naples Institute by comparison with natural seawater. The animals kept well in this artificial seawater, if not quite as long as in natural seawater. There was no effect on phototaxis on transferring the nauplii into artificial seawater. That is: the time elapsing until the reverse of orientation under the influence of a certain source of light was neither shorter nor longer than in control animals kept in natural seawater. The artificial seawater was therefore in its influence on phototaxis equivalent to natural seawater.

When the animals were transferred into pure isotonic $\mathrm{NaCl}$ solution, negative larvae became positive, positive larvae more positive. Moreover, positive animals would not become negative again, even under the influence of full sunlight, including the ultra-violet rays. They died after a short time through 
the action of the ultra-violet rays, sinking down on the positive side of the glass. They had apparently lost the possibility of negative orientation. The same effect could also be obtained by adding to the $\mathrm{NaCl}$ solution pure natural seawater in the proportion of $1: 1$; in this case the animals were less affected by the solution, while in pure $\mathrm{NaCl}$ they sometimes died after a quarter or a half-hour. Added to natural seawater in small doses, $\mathrm{NaCl}$ solution accelerates the positive and retards the negative reaction at a degree depending on the value of the quotient $\frac{p}{n}$ (Lichtstimmung). Correspondingly, the threshold concentration required to bring about positive reaction in negative animals is different. Leaving $\mathrm{NaCl}$ out of artificial seawater has an effect contrary to that obtained by adding it to pure seawater. It increases the negative and diminishes the positive reaction. The best solution proved to be one containing about two-thirds of the ordinary amount of $\mathrm{NaCl}$. Lower concentrations were harmful. The result obtained pointed to the probability, that there were other salts in the solution antagonistic to $\mathrm{NaCl}$, whose effect predominated when $\mathrm{NaCl}$ was diminished.

Potassium chloride had an effect similar to, but considerably weaker than that exhibited by sodium chloride. Pure isotonic $\mathrm{KCl}$ solution killed the animals instantly and if added to natural seawater in proportions above 1:150 proved to be poisonous also. If the concentration is slightly weaker than $1: 150$, positive reaction is increased, negative reaction diminished, but I never saw large quantities of animals become instantly positive, as they did after a sufficient addition of isotonic $\mathrm{NaCl}$. $\mathrm{KCl}$ does not change the effect of pure $\mathrm{NaCl}$ solution if added in the proportion prevailing in natural seawater, nor does its omission from artificial seawater cause any alteration of the reaction.

Calcium chloride belongs to the same group as the two first mentioned salts. Pure 0.65 molecular solution has a toxic effect. If added to natural seawater in the proportion of 1-15 it inhibits negative reaction in a very short time, both in negative and in positive animals about to become negative. But it has the singular secondary effect of apparently paralyzing the larvae. The 
rhythm of the locomotor movements is retarded and the orientation to light is at length totally abolished, causing the animals to swim about unoriented. It would therefore be hardly appropriate to attribute a positivating influence to $\mathrm{CaCl}_{2}$. In a long standing tube with the source of light high above it, when normal animals would gather near the surface, animals in the $\mathrm{CaCl}_{2}$ solution are equally distributed throughout the entire length of the tube; the reaction to changes of intensity is considerably diminished or disappears entirely. Nevertheless, the animals live indefinitely in such a solution and swim about continuously. With $\mathrm{CaCl}_{2}$ also there was no effect when it was added to isotonic $\mathrm{NaCl}$ or omitted from artificial seawater.

As in the two last mentioned salts, so in magnesium chloride, the pure solution was toxic. I may recall the fact that in pure $\mathrm{NaCl}$ solution the nauplii would not become negative. If, however, $\mathrm{MgCl}_{2}$ or $\mathrm{MgSO}_{4}$ was added in the ordinary proportion of artificial seawater to pure $\mathrm{NaCl}$ solution, the retarded negative reaction would be called forth instantly, provided the light was strong enough. There is no appreciable difference in the rate of negativation under the influence of a given source of light between larvae in natural seawater and in the mixture of sodium and magnesium solution. Apparently magnesium has the opposite or antagonistic effect to sodium. It was now the question, whether magnesium has in itself a negativating effect, analogous to increase in temperature, or whether it merely compensates the effect of sodium (potassium) if present in a definite proportion to these salts. This question is answered by experiments, in which magnesium chloride or sulphate was added to natural seawater. In this case there is no negativating effect whatever, even with the strongest concentration the larvae could permanently stand $(1: 25)$. We are therefore justified in assuming that magnesium acts only as a compensation to the positivating influence of sodium but possesses no negativating influence. This is confirmed by the experiments with artificial seawater without magnesium. If the solution contains no magnesium, the effect is similar to that of pure sodium chloride, but the animals would keep no better than in pure $\mathrm{NaCl}$ solution. 
When natural seawater is added to the artificial magnesium-free seawater in a proportion of $3: 1$, the animals would live in this solution for some hours without becoming negative, save in a few exceptional cases. Decrease of magnesium has therefore the same effect as increase of sodium or potassium. In other words, for a normal production of light reactions it is necessary to have the correct proportion of sodium (potassium) on one side and magnesium on the other. In my experiments magnesium chloride proved slightly more effective than the sulphate.

Lastly, among the constituents of seawater I have to mention the alkali content. With the concentration prevailing in natural seawater no effect could be detected. When all alkali was left out of artificial seawater or added to pure $\mathrm{NaCl}$ solution, the reaction of the nauplii was not changed. A stronger concentration of OH-ions, however, (about 2 cc. $\frac{\mathrm{N}}{10} \mathrm{NaOH}$ in 100 cc. of seawater) had a visible negativating influence; ammonia was still more effective (about $1 \mathrm{cc} \cdot \frac{\mathrm{N}}{10} \mathrm{NH}_{3}$ in 100 cc. of seawater), due according to the experiments on sea-urchin eggs, to its permeating more quickly into the protoplasm. The reaction of the seawater had in both cases become strongly alkaline to neutral red. Both alkalies in the concentration mentioned would quickly make positive animals negative and prevent negative animals from becoming positive, even in very weak light.

I believed it to be of interest, with reference to the papers by Loeb mentioned above, to see whether acids would have the opposite effect. Contrary to the negative results that author obtained in marine forms of America, I found that the mineral acids $\mathrm{HCl}, \mathrm{H}_{2} \mathrm{SO}_{4}$ and $\mathrm{HNO}_{3}$ had a positivating effect, but that the effective concentration had very narrow limits. Only such concentration would prove successful, as gave neutral red a slight pink hue, natural seawater giving a reddish yellow color. Slightly higher concentrations killed the animals, lower ones had no effect. In acetic acid and $\mathrm{Co}_{2} \mathrm{I}$ saw no positivating influence nor was it very strong in the mineral acids mentioned.

Lack of oxygen has a very strong effect on phototaxis. If nauplii are put in seawater which has been evacuated for a 
few minutes by means of a filter pump (at about $300 \mathrm{~mm}$. of mercury pressure) they become positive immediately and remain so as long as they are left in the water. Transferred into fresh water they become negative again with equal velocity. This is one of the strongest and most striking reactions. Traces of metal acted in the reverse sense. If traces of copper $\left(\mathrm{Cu}_{2}\right.$ concentration $=2.10^{-5}$ ) are added to the seawater, all positive animals soon become negative and remain so. This effect is in accordance with the experiments on oxydation in the sea-urchin egg, where traces of metal also acted in the same sense as alkali and increase in temperature. I did not, however, repeat this experiment more than three or four times with different animals.

I found no marked positivating or negativating effect with narcotics, of which alcohol, ether and chloroform were tried, though the tendency was rather in the former direction. They would, however, disorient the animals, like calcium, causing them not to assemble at the poles of the vessel but to swim about freely in all directions.

Experiments with potassium cyanide, strychnine, oxygen and hydrogen peroxyde met with no success. Finally, I made experiments with changes in concentration. The result was the same observed by Loeb with Copepods: increase in concentration had a positivating effect, decrease a negativating one. My best hypertonic solution contained 1 gram of pure crystalline $\mathrm{NaCl}$ in $100 \mathrm{cc}$. of seawater. The effect was very strong. To exclude the possibility, that the higher concentration of $\mathrm{Na}$ ions was responsible for this result, I made a hypertonic solution of magnesium chloride which could in no way be suspected of a specific positivating salt action. The positivating effect was less marked but sufficiently evident. I am therefore justified in concluding, that hypertonicity alone has a positivating influence, though in the first case it may have been seconded by the effect of the increase in Nations. Increase in concentration has the opposite effect. Seawater mixed with fresh water in the proportion of $7: 1$ quickly makes positive animals negative and prevents negative ones from becoming positive. 
Unlike the Californian form used by Loeb my nauplii showed no lack of precision in their response to changes of concentration.

To sum up, we may repeat the names of all the agents experimented with, classed in three groups. The first contains those with a positivating effect, including sodium, potassium, acids, deoxygenated seawater, hypertonic seawater and decrease of temperature. The second contains those with a negativating effect, including certain visible and invisible light rays, alkali, traces of metal, hypotonic solution and increase of temperature. The third contains narcotics, causing the animals to lose their sensitiveness to light and including calcium, alcohol, ether and chloroform.

I will conclude the account of my work on Balanus nauplii by a number of experiments made to test the influence of various dyes on phototaxis. The larvae were transferred for some time into solutions of stains and then exposed to light of different wave lengths either in these solutions or in pure seawater. I tried methylene blue, eosine, erythrosine, Bismarck brown, methyl green, neutral red and orange. The effect of staining on the light reactions was ascertained by comparison with unstained animals, special care being taken to keep both stained and unstained animals under equal conditions. The water was always taken out of the same aquarium for both sets of animals. The only variable was the addition of the dye.

I soon observed that animals which had been stained in Bismarck brown or methylene blue became negative more quickly than unstained larvae when exposed to white light. It was sufficient for the animals to remain for one or two hours in a solution with just a shade of brown or blue color, to obtain this result and the same effect was reached when the animals were put into the color solution and exposed to light at once. With eosine the effect was very slight and not always observable and with the rest of the dyes no effect was seen at all.

I then exposed animals stained brown or blue to strong artificial light made monochromatic by color solutions. I used a solution of potassium monochromate for the yellow and a solu- 
tion of copper acetate with addition of methyl green and lithium carmine for the blue filter. In both colors the animals stained with Bismarck brown became negative first, those stained with methylene blue second and the unstained ones last. I was therefore not able to state that the complementary colors chiefly absorbed by the dye (yellow for the methylene blue and blue for the Bismarck brown) had a stronger effect than those of the same color. This is, however, what ought to have been the case, if I had succeeded in producing a 'sensitisation.' Moreover, the difference in the velocity of negativation between stained and unstained animals was not considerable, the negativating effect of the dyes being very small in comparison to most of the agents mentioned in this paper. The effect of heat was not, however, to be made responsible for the result; special measurements showed that the differences never exceeded 0.4 to 0.5 of a degree (C.), the water being warmer sometimes in the colored and sometimes in the uncolored water. The poisonous effect of the dyes made it impossible to use higher concentrations. All these observations indicated the probability that the effect of methylene blue and Bismarck brown had nothing to do with their color but with their containing some chemical agent, which would make the animals negative in small concentrations and kill them in stronger ones. It is well known that methylene blue is poisonous for living protoplasm, especially in the light, and the same is true, according to Straub, for eosine, which is said to form a hypothetical poisonous eosine peroxide under the influence of light. In my experiments all the dyes mentioned proved to be toxic even in the dark in concentrations slightly higher than those used for my purposes. To obtain a final answer to the question at issue I made use of the reaction to changes of intensity of illumination, mentioned in the beginning of this paper. Three glass tubes with brown, blue and unstained nauplii were made to adapt themselves to weak light coming from above. After some time, the strong monochromatic light was turned on, the source of light being at a level with the surface of the water in the tubes. The nauplii sank every time with equal velocity in all three tubes, whether I 
turned on the yellow or the blue light. I concluded that there is no sensitisation of the protoplasm of eye or body of the nauplii by the dyes used and that their negativating influence is probably due to a non-photochemical effect on the protoplasm.

Concluding from the information gained by my experiments, on the behavior of free Balanus larvae under normal conditions, it may be supposed that they react very similarly to the other planktonic forms investigated by the present author. After hatching, the larvae swim towards the surface, the strong increase of light causing them to sink down again very soon by inhibition of their locomotion. Their movements will consist in a continuous alternation of sinking and rising ("periodical locomotion,' Ewald '10) caused by successive inhibition and stimulation, without ever necessitating the taking place of negative reaction. This reaction probably constitutes an artificial product of the laboratory. The 'periodical locomotion' as described in the paper referred to above, causes the animals to maintain themselves in an area of equal illumination throughout the day, taking them gradually up in the evening and down in the morning. In the evening, decrease of illumination will slowly shift the position where inhibition due to prolonged upward locomotion takes place, nearer and nearer the surface, while the reverse is the case for the morning. It is thus unnecessary to assume that the animals constantly change between positive and negative reaction, as was supposed by Loeb. The eminent usefulness of this mechanism is shown by the experiments demonstrating the strong deleterious effect of the light rays of short wave lengths on the nauplii.

\section{SUMMARY}

\section{Effects of light}

1. The nauplii of Balanus perforatus show the same reactions to changes of intensity of locomotion, as that found by the author in Cladocera and Copepods. Increase of illumination causes inhibition of locomotion, preceded by a slight acceleration; the result is a sinking. Decrease of illumination causes 
acceleration of locomotion. Within limits, the absolute intensity of illumination does not affect these reactions.

2. It was found that light of different wave lengths influenced these reactions in a different way. Green and yellow-green have the strongest effect; blue-green, blue, yellow, violet and red follow in the order named.

3. The same order of efficiency is found, when the orienting power of different parts of the spectrum is tested. The positive animals collect in the green on the side near the light, the negative animals in the red and violet on the far side.

4 . The velocity with which positive animals become negative is also different for different parts of the spectrum, but in a different order. Balanus larvae became negative most quickly in the violet and in the green, less quickly in the blue and the yellow and hardly at all in the red.

5. The ultra-violet rays have the most strongly deleterious effect on the larvae. The violet rays follow next, then the green and the blue, while the red rays of the intensities tested had no harmful effect.

\section{Effects of temperature}

6. Increase of temperature made positive animals negative and negative animals more strongly negative; decrease of temperature made negative animals positive and positive animals more strongly positive.

\section{Effects of the salts of the seawater in isotonic solutions}

7. Isotonic sodium chloride solution, pure or added to natural seawater, makes negative animals positive and positive animals more positive. If sufficiently in excess of the other salts, it inhibits negative reaction entirely.

8. Isotonic potassium chloride solution, added to natural seawater, acts in the same direction, though less effectively.

9. Isotonic calcium chloride solution, added to natural seawater, makes the larvae lose their power of reaction to light stimuli, causing them to swim about at random without negative or positive orientation. 
10. Magnesium chloride or sulphate solution acts as an antagonist to sodium. Added in the proportion prevailing in natural seawater to pure $\mathrm{NaCl}$ solution, $\mathrm{MgCl}_{2}$ brings about the negative reaction which is suspended in pure $\mathrm{NaCl}$ solution. There is no difference in response to photic stimuli between larvae in the sodium magnesium mixture and in pure seawater. For a normal production of light reactions it is necessary to have the correct proportion of sodium on one side and magnesium on the other.

\section{$I V$. Other chemical effects}

11. Sodium hydrate or ammonia above a certain concentration had a strong negativating effect.

12. The mineral acids $\mathrm{HCl}, \mathrm{H}_{2} \mathrm{SO}_{4}$ and $\mathrm{HNO}_{3}$ in a certain concentration had the opposite effect. Acetic *acid and carbonic acid had no effect.

13. Lack of oxygen (brought about by evacuating the seawater) had a very strong positivating effect.

14. Traces of copper had a negativating effect.

15. Alcohol, chloroform and ether caused the animals to lose their reactions to light.

\section{$V$. Effects of changes in concentration}

16. Hypertonic solutions of $\mathrm{NaCl}$ or $\mathrm{MgCl}_{2}$ had a strong positivating effect, hypotonic solution of $\mathrm{NaCl}$ had an equally obvious negativating effect.

VI. Effect of stains

17. Staining nauplii with Bismarck brown or methylene blue had a weak negativating influence, due, however, not to a specific color effect ('sensitisation') but to certain chemical contents of the stain. Other stains tried had no effect whatever. 


\section{BIBLIOGRAPHY}

Ewald, Wolfgang F. 1910 Utber Orientierung, Lokomotion und Lichtreaktionen einiger Cladoceren und deren Bedeutung für die Theorie der Tropismen. Biolog. Centralblatt, vol. 30 .

Hertel, E. 1905 Über physiologische Wirkungen der Strahlen verschiedener Wellenlänge, Zeitschr. f. allg. Physiol., vol. 5.

1904 Über die Beeinfüssung des Organismus dureh Licht, speciell d. chemisch wirksamen Strahlen. Zeitschr. f. allg. Physiol., vol. 4.

Hess, C. 1911 Der Gesichtssinn. Wintersteins Handbuch d.vergleichenden Physiologie, vol. 4.

Holmes, S. J. 1905 The reactions of Ranatra to light. Jour. Comp. Neur., vol. 15 .

LoeB, JACQUes 1906 The dynamies of living matter. New York.

MASsART, J. 1888 and 1891 Recherches sur les organismes inférieurs. Bull. Acad. Belg., ser. 3, t. 16 and 22.

MAST, O. 1911 Light and the behavior of organisms.

Rothert, W. 1904 Über die Einwirkung des Athers und Chloroforms auf die Reizbewegungen der Mikroorganismen. Jahrb. fur wissensch. Botanik, vol. 39.

Straub 1904 Über den Chemismus der Wirkung belichteter Eosinlösung auf oxydable Substanzen. Arch. f. experim. Pathologie, vol. 51 .

Strassburger, E. 1878 Wirkungen des Lichts und der Wärme auf Schwärmsporen. Jen. Zeitschr., N. F. vol. 12.

WarbURG, O. 1908-1910 Über die Oxydationen im Ei, I, II, III. HoppeSeylers Zeitschr. f. physiol. Chemie, vol. 57, vol. 60 and vol. 66. 